\title{
Numerical Simulation of the Solitary Waves Propagation and Run-up in Shallow Water
}

\author{
A. Yu. Belokon ${ }^{\bowtie}$, S. Yu. Mikhailichenko \\ Marine Hydrophysical Institute of RAS, Sevastopol, Russian Federation \\ 凶aleksa.44.33@gmail.com
}

\begin{abstract}
Purpose. The paper is aimed at investigating the propagation of solitons in a shallow basin, assessing the nonlinear effects resulting from the wave run-up on a gentle coast, and at comparing the estimates obtained using different numerical models with the available analytical dependencies.

Methods and Results. The results of numerical simulations carried out using two nonlinear models of long waves (the author's model and the Simulating WAves till SHore (SWASH) one) are represented in the paper. The solitary wave profiles were obtained during its propagation in the part of a basin with constant depth conjugated with the inclined bottom. The process of a wave run-up on the coast was simulated using the algorithm of fluid movement along a dry coast. It is shown that when a soliton propagates in the basin part with constant depth, the nonlinearity effects are manifested in deformation of a wave profile. In other words, increase of the wave initial amplitude and the distance traveled by a wave is accompanied by growth of the wave front slope steepness. This, in its turn, leads to increase of a splash when the waves run-up on the coast. The estimates of the run-up heights resulted from different numerical models are in good agreement.

Conclusions. The calculated values of the maximum wave run-up on the coast for the non-deformed waves, the length of which is equal to that of the traversed path, are close to the estimates obtained analytically. For the waves with the deformed profile, the front slope steepness of which increases with propagation over long distances, the run-up heights increase with growth of the wave initial amplitude. In such a case, it is desirable to replace the analytical estimates with the numerical ones. The run-up height of the deformed waves can exceed the wave initial amplitude by four or more times. The results obtained in this study can be useful in projecting the coastal protection constructions with the regard for preserving the coastal ecology and economy.
\end{abstract}

Keywords: numerical simulation, solitons, solitary waves, nonlinear effects, run-up, hydrodynamic model SWASH

Acknowledgment: the investigation was carried out within the framework of the state task on theme No. 0555-2021-0005 "Complex interdisciplinary investigations of the oceanologic processes conditioning functioning and evolution of the Black and Azov seas' ecosystems of the coastal zones”.

For citation: Belokon, A.Yu. and Mikhailichenko, S.Yu., 2021. Numerical Simulation of the Solitary Waves Propagation and Run-up in Shallow Water. Physical Oceanography, [e-journal] 28(6), pp. 691-701. doi:10.22449/1573-160X-2021-6-691-701

DOI: 10.22449/1573-160X-2021-6-691-701

(C) A. Yu. Belokon, S. Yu. Mikhailichenko, 2021

(C) Physical Oceanography, 2021

\section{Introduction}

The study of the solitary waves' propagation in the shallow water, followed by a run-up onto the coastal slope, is a practically significant task. Such processes can be observed, first of all, when tsunami waves, as well as tidal and ship waves, approach the coast. In the open ocean, these waves have a small amplitude and do not pose a particular danger, however, as they approach the coast, their propagation velocity decreases, and the height increases. When approaching the coast at 
a certain stage of wave deformation in shallow water, wind waves transform into the solitary waves [1]. In [2], it was suggested that the wind solitons are one of the possible causes for the formation of rogue waves in a shallow sea. Frequent observation of solitons in the shallow sea zones was noted in [3]. In the shallow water, the impact of non-linear effects increases, which entails an increase in the run-up heights and leads to a destructive effect on the coast. The main danger of such waves is that they can be amplified by other phenomena, for example, tides, while penetrating long distances into the interior of the coast [4].

The analytical solution of the nonlinear shallow water equations on a flat slope was found for the first time in [5]. The approach used there was further developed in a number of works by various authors [6-13]. In [7, 8], analytical estimates were obtained for determining the heights of the run-up of non-breaking waves on a flat slope, which are in fairly good agreement with the experimental data. Comparison of numerical calculations of wave run-up on a flat slope with experimental data and analytical estimates of various authors was carried out in [9]. Experimental studies are described in [10-12].

The main attention in the abovementioned works is paid to the determination of the maximum wave run-up depending on its amplitude and the shore inclination angle. At the same time, it is known that the height of the wave run-up to the coast substantially depends on the steepness of the front slope of the wave [13, 14]. The waves, penetrating into the shallow water, become "sensitive" to a decrease in depth, and, as a result, come to the shore deformed: their front slope becomes steep, which can eventually lead to extreme run-up. In $[13,15]$ it was demonstrated that the waves with a steep front slope can penetrate deep into the land for greater distances than the waves with a symmetric profile. Within the framework of the theory proposed in [13], the role of the wave steepness as a determining parameter for calculating the height of the tsunami wave run-up becomes clear. In [16], an appropriate formula for the maximum run-up height was proposed, taking into account the steepness of the wave front.

In this work, we continue to study the run-up of nonlinearly deformed waves on a gentle slope. A numerical simulation of the solitary wave propagation in a model basin, consisting of a section of constant depth connected to an inclined bottom, followed by a wave run up to the shore, has been carried out. The calculations were carried out using two nonlinear numerical models the model from [14] and the Simulating WAves till SHore (SWASH) model *. The influence of nonlinearity on the deformation of the wave was studied in the section of constant depth. Estimates for the heights of the wave splash when running up a gentle coast are obtained. The results obtained numerically within the framework of these models were compared with each other and with analytical dependences obtained by other authors.

* The SWASH team, 2012. SWASH (7.01). [computer program] Delft University of Technology. [online] Available at: https://swash.sourceforge.io [Accessed: 31 November 2021]. 


\section{Mathematical problem statement}

We consider the problem of a solitary surface wave propagation in a basin with a slope followed by a run-up to the coastal slope. In Fig. 1 a model basin with a depth $H(x)$, which consists of a section of constant depth $H_{0}$ of length $L$, connected to an inclined section with a bottom slope $\alpha$.

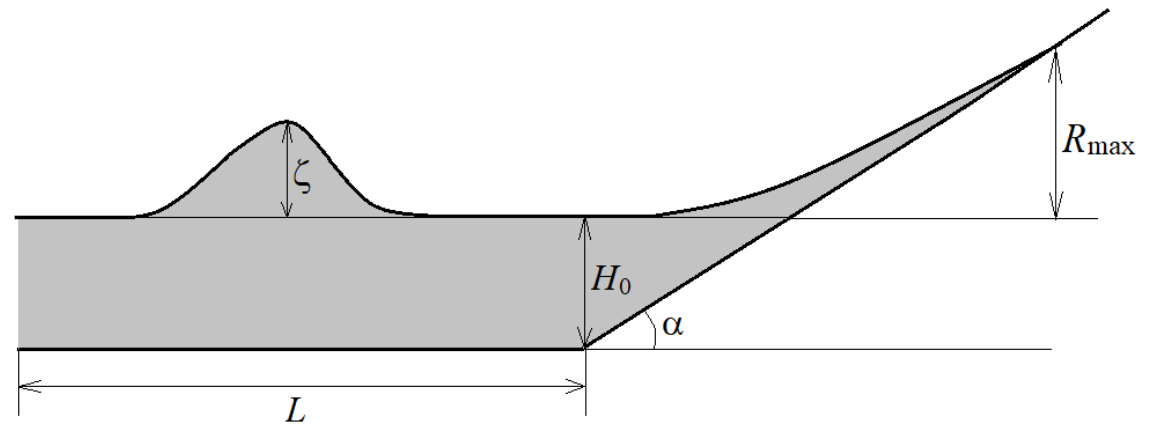

F i g. 1. Scheme of a solitary surface wave propagation in a basin followed by run-up on the coastal slope

The process of wave propagation in the model basin was described using a nonlinear model of long surface waves, which operates with a depth-averaged horizontal velocity and sea level displacement [17]:

$$
\frac{\partial u}{\partial t}+u \frac{\partial u}{\partial x}=-g \frac{\partial \zeta}{\partial x}, \quad \frac{\partial \zeta}{\partial t}+\frac{\partial(u D)}{\partial x}=0,
$$

where $u=u(x, t)$ is a horizontal velocity averaged over the basin depth; $t$ is time; $x$ is a longitudinal coordinate; $g$ is the gravitational acceleration; $\zeta=\zeta(x, t)$ is a sea level displacement averaged along the cross section of the basin; $D(x, t)=H(x)+\zeta(x, t)$ is dynamic (full) depth of the basin.

The initial conditions assume that at $t=0$ the liquid in the basin is at rest:

$$
u=0, \quad \zeta=0 \quad(t=0) .
$$

The entrance of a solitary wave of initial height $a_{0}$ into the basin was modeled using boundary conditions. At the liquid boundary $x=0$, the entrance of a solitary wave of initial height $a_{0}$ into the basin was specified as a time series of sea level displacement values corresponding to the Korteweg-de Vries soliton profile:

$$
\zeta=a_{0} \operatorname{sech}^{2}\left(\frac{1}{4} \sqrt{\frac{3 a_{0}}{H_{0}}} \frac{(\lambda-2 C t)}{H_{0}}\right) \quad(x=0,0<t \leq T),
$$

where $T=\lambda / C$ is a time of the wave passage of $x=0 ; \lambda=4 H_{0} \sqrt{H_{0}\left(3 a_{0}\right) \operatorname{arch}} \sqrt{20}$ is a characteristic width of a solitary wave profile at $a_{0} / 20$ height $[7,8]$; $C=C(0)=\sqrt{g H_{0}}$ is a wave phase velocity at the basin entrance. The period $T$ is "conditional" [1] period of a solitary wave, which characterizes in this work PHYSICAL OCEANOGRAPHY VOL. 28 ISS. 6 (2021) 
the time during which the soliton passes through the section located between the points of the wavefront at a height of $1 / 20$ of the amplitude, through the section $x=0$. This parameter is determined by the length and velocity of the solitary wave propagation. The soliton length as the wave width at a height of $1 / 20$ of the initial amplitude was chosen in this work in order to accurately set the wave profile and preserve its shape when entering the computational domain.

From the moment of the wave complete penetration of the into the channel (at $t=T$ ) at the liquid boundary $x=0$, the condition of wave generation (3) is replaced by the condition of free wave exit:

$$
\frac{\partial u}{\partial t}-C \frac{\partial u}{\partial x}=0(x=0, t>T) .
$$

The initial boundary value problem (1) - (4) was solved numerically using the finite difference method using an explicitly implicit first order approximation scheme in time. The flooding - drainage process was modeled using the algorithm described in [18]. The calculations were carried out on a grid with $\Delta x=1$ spatial resolution and with $\Delta \mathrm{t}=0.01 \mathrm{~s}$ time step.

Some of the numerical experiments were carried out using the SWASH hydrodynamic model, which is based on nonlinear shallow water equations. Unlike [14], this model can take into account non-hydrostatic effects. For comparability of the results of the two models, these effects were not included in SWASH.

\section{Study results and their discussion}

We consider the propagation of a solitary surface wave, the shape of which is specified in the form of a soliton (3). The initial wave amplitude is $0.2 \mathrm{~m}$, its length is $\sim 1000 \mathrm{~m}$ (at a height of $1 / 20$ of the amplitude). In Fig. 2 the sea level distributions during the wave propagation along a section of constant depth $H_{0}=20 \mathrm{~m}$, the length of which is $15 \mathrm{~km}$, are shown. The waveforms at different times, obtained within the framework of the nonlinear long wave model described by formulas (1) - (4), and the profiles calculated using the SWASH model, are presented.

In Fig. 2 it can be seen how the wave that entered the computational domain transforms over time. The front slope of the wave becomes steep, while its length and initial amplitude remain unchanged. With the further propagation of the wave along a section of constant depth, its front slope will become steeper until the breaking occurs. In [7], the breaking criterion is determined by the analytical formula

$$
\frac{a_{0}}{H_{0}}=0.8183(\operatorname{ctg} \alpha)^{-\frac{10}{9}} .
$$

However, this equation is valid for those waves that, when approaching the slope, have a symmetrical shape. The criterion for the wave breaking in the model of nonlinear long waves from [14] was the condition under which the orbital velocity of a particle on the wave crest becomes greater than the wave phase velocity, i.e. $\partial \zeta / \partial t \geq C$. 


\section{○ long wave nonlinear model $\quad$ model SWASH}
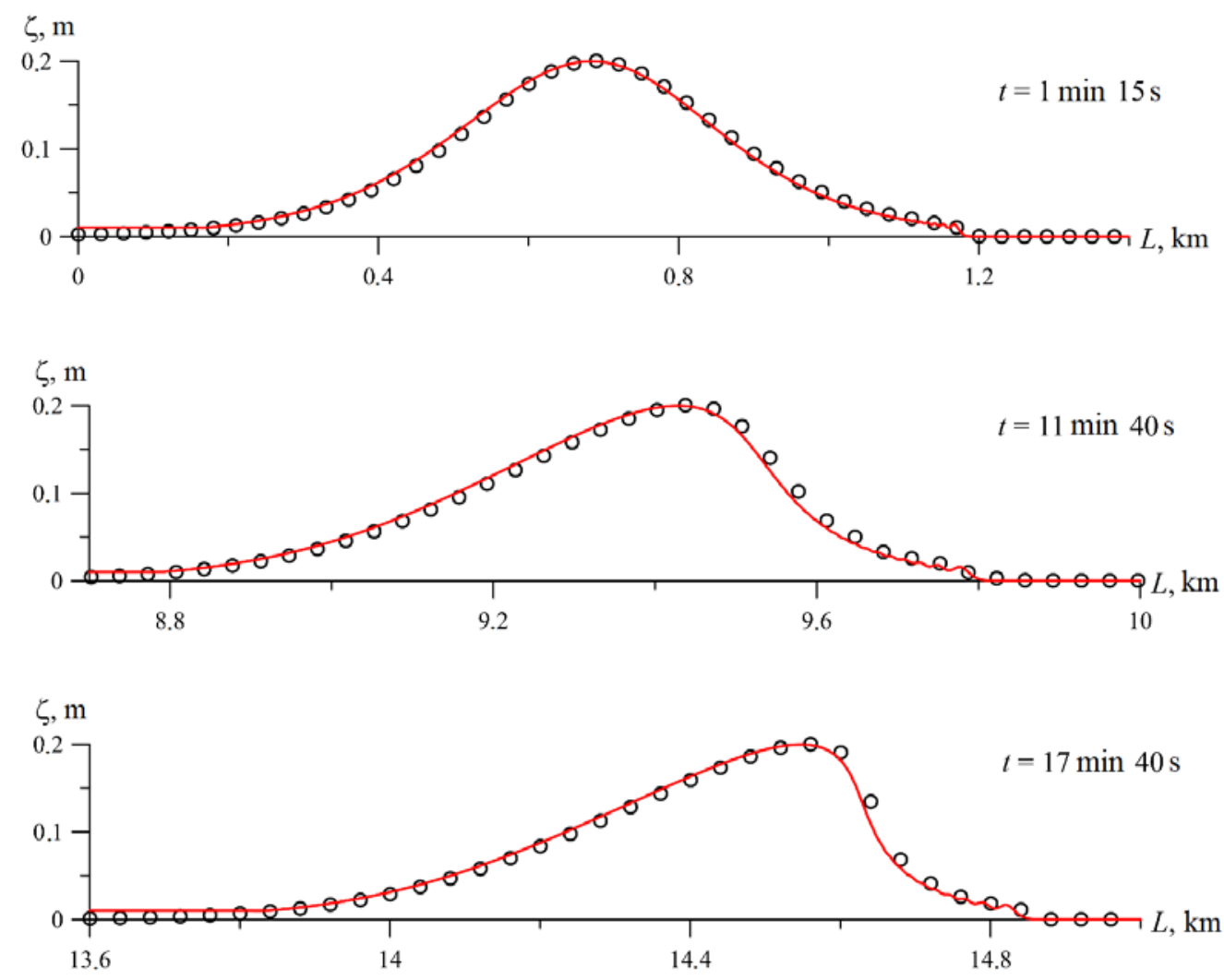

F i g. 2. Soliton propagation in a basin of constant depth at different time moments. The wave initial amplitude $a_{0}=0.2 \mathrm{~m}$, the basin depth $H_{0}=20 \mathrm{~m}$

Wave breaking in the SWASH model was taken into account by the following criterion:

$$
\frac{\partial \zeta}{\partial t}>\beta \sqrt{g D},
$$

where $\beta=0.6$ is the maximum wave steepness.

After passing a section of constant depth, the wave continues its movement on an inclined section, and then along a dry coast. In Fig. 3 the position of the shoreline at the moment of the maximum wave run-up to the coast and the maximum drainage of the bottom is shown. The curves, calculated within the framework of the nonlinear model of long waves and using the SWASH model, which are in good agreement, are given. A slight excess of the run-up for the curve obtained within the framework of the nonlinear long-wave model is observed. Thus, the height of the wave run-up exceeds the initial wave amplitude by 4.4 times. The drop in sea level during the run-down was $\sim 0.5$ of the initial wave amplitude. 


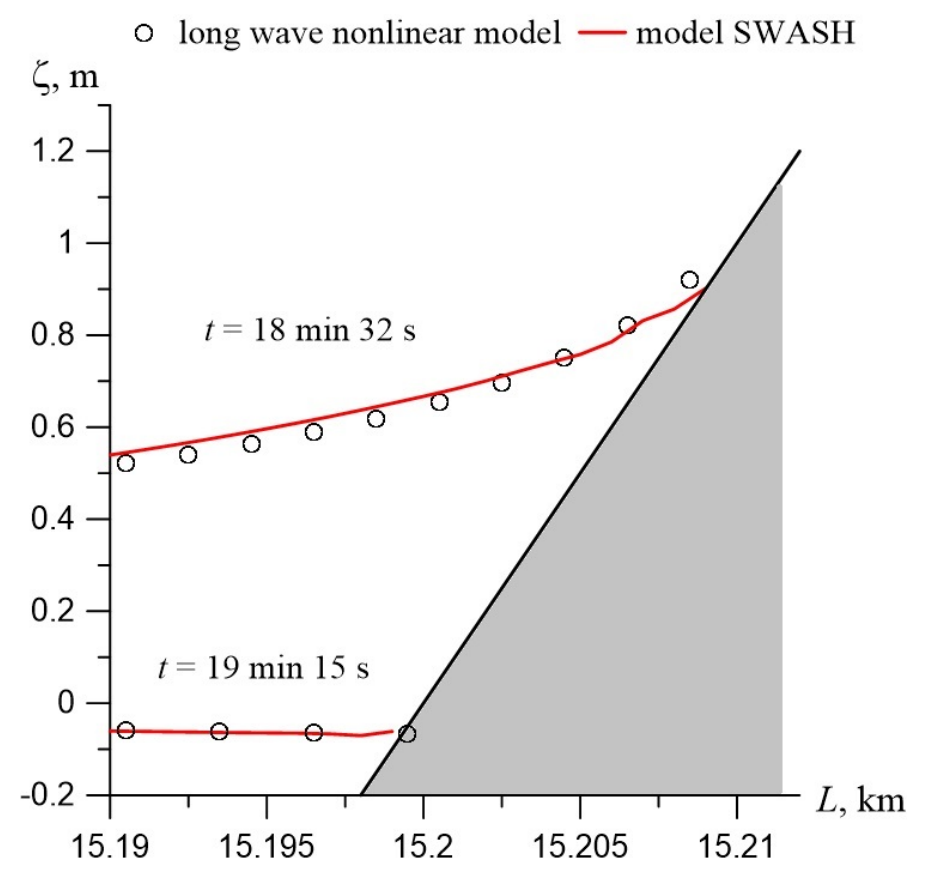

F i g. 3. Sea level at the moment of the maximum wave run-up on the coast and subsequent rundown. The wave initial amplitude $a_{0}=0.2 \mathrm{~m}$, the basin depth $H_{0}=20 \mathrm{~m}$, the length of the constant depth section $L=15 \mathrm{~km}$, the bottom slope tg $\alpha=0.1$

Reflecting from the coast, the wave moves in the opposite direction. The reflected wave is demonstrated in Fig. 4, where it can be seen that the solitary wave, after the run-up onto the shore, is transformed into an alternating wave.

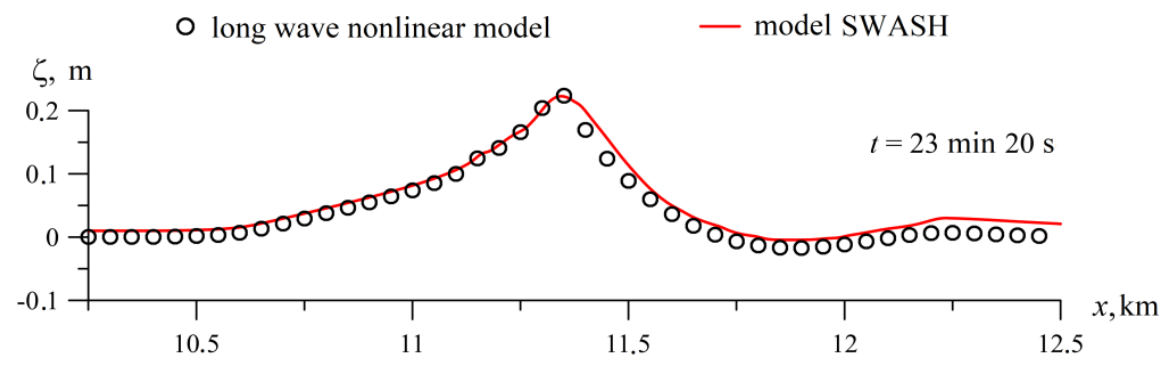

F i g. 4. The reflected wave profile while its propagation and subsequent run-up on the coast. The wave initial height $a_{0}=0.2 \mathrm{~m}$, the basin depth $H_{0}=20 \mathrm{~m}$, the length of the constant depth section $L=15 \mathrm{~km}$, the bottom slope $\operatorname{tg} \alpha=0.1$

Profile curves in Fig. 2 demonstrate that the greater the distance over which the wave propagates, the stronger the effects of nonlinearity appear, which leads to an increase in the steepness of the wave front slope. We study the height of the runup of solitons on the coastal slope depending on the length of the section $L$ of constant depth. In Fig. 5 the estimates of solitary waves' splashes of various amplitudes, calculated within the framework of both models, are shown. It can be 
seen that for undeformed waves (at $L / \lambda=1$ ), the ratio of the run-up height to the initial amplitude is 3.2 at $a_{0}=0.2 \mathrm{~m}, 3.5$ at $a_{0}=0.3 \mathrm{~m}, 3.9$ at $a_{0}=0.5 \mathrm{~m}$. With an increase in the length of a section of constant depth, the process of wave propagation is accompanied by an increase in the steepness of the wave front slope, which leads to a splash increase when the waves run-up to the shore.
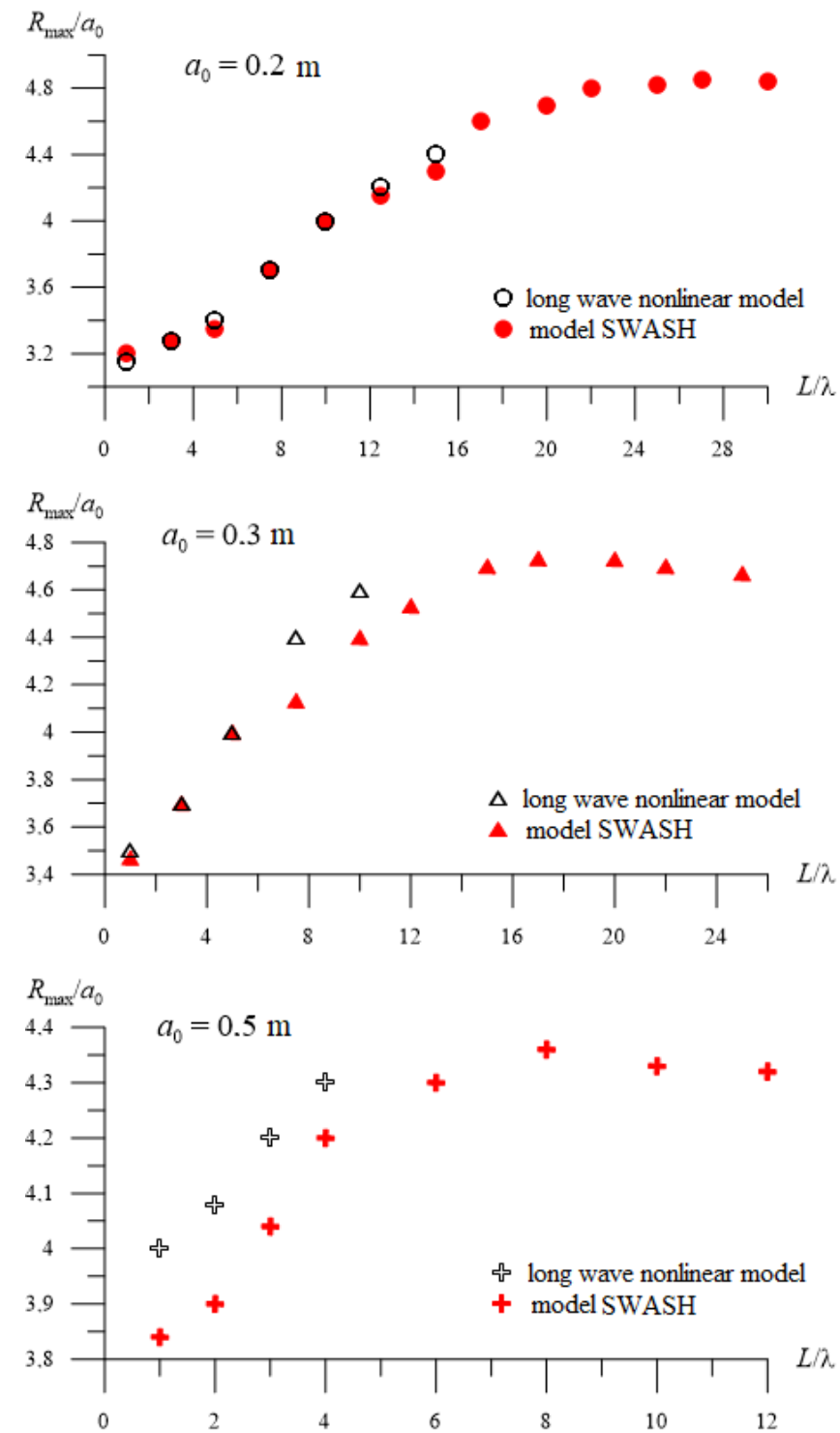

F i g. 5. Estimates of relative heights of the solitary wave run-ups on the coast depending on the length of the basin part with constant depth for different wave initial amplitudes. The basin depth $H_{0}=20 \mathrm{~m}$, the bottom slope tg $\alpha=0.1$

In Fig. 5, the estimates obtained using the nonlinear long wave model (1) - (4) represent the run-up heights of non-breaking waves. When the maximum steepness of the front slope is reached, the wave breaks down, and further numerical 
experiments are possible only in the SWASH model, which assumes the calculation of the propagation and run-up of breaking waves on the coast. It can be seen (Fig. 5) how, after the wave breaks, the run-up height reaches its maximum, and then begins to decrease due to energy dissipation during the breaking. An increase in the initial wave amplitude also leads to an increase in the splash. At the same relative distance $L / \lambda$, the run-up heights turn out to be the largest for waves with larger initial amplitudes. At the same time, the larger the amplitude of the solitary wave is, the smaller the characteristic width of the wave profile is, which leads to a more rapid onset of wave breaking and energy dissipation. Thus, for deformed non-breaking waves of small amplitude $\left(a_{0}=0.2 \mathrm{~m}\right)$, the height of the run-up onto the shore increases by 4.4 times, for $a_{0}=0.3 \mathrm{~m}$ - by $4.5-4.6$ times, for waves of large amplitude $\left(a_{0}=0.5 \mathrm{~m}\right)-4.2-4.3$ times. Further studies within the framework of the SWASH model showed that the maximum height of the breaking wave runup exceeded the initial wave amplitude by 4.85 times at $a_{0}=0.2 \mathrm{~m}$, by 4.73 times at $a_{0}=0.3 \mathrm{~m}$, by 4.36 times at $a_{0}=0.5 \mathrm{~m}$.

The numerical estimates obtained using these models showed good agreement. The difference in the run-up heights for large amplitudes is associated with the use of different algorithms in the models when calculating the run-up.

The analysis of comparison of the results obtained numerically and analytically is carried out. In [7], the following expression was proposed for determining the run-up height:

$$
\frac{R_{\max }}{H_{0}}=2.831 \sqrt{\operatorname{ctg} \alpha}\left(\frac{a_{0}}{H_{0}}\right)^{\frac{5}{4}} .
$$

In [16], the dependence of the run-up height on the wave steepness was obtained:

$$
R_{\max }=R_{0}\left(\frac{\mathrm{s}}{\mathrm{s}_{0}}\right)^{0.42},
$$

where $R_{0}$ is run-up height of undeformed wave; $s$ is the maximum steepness of the nonlinearly deformed wave; $s_{0}$ is the maximum steepness of undeformed wave.

In Fig. 6 the analytical curve calculated by the formula (6) for the height of the waves with a symmetrical profile is shown. It also presents analytical dependences found by formula (7) for the waves with a nonlinearly deformed profile, and numerical estimates for non-breaking waves obtained within the framework of the models described above. It can be seen that the numerical estimates of the splashes are close in magnitude to the analytical estimates. However, with an increase in the distance over which the wave propagates, the heights of the wave run-up onto the coast also increase. Thus, analytical estimates can provide a good quantitative description for the waves whose wavelength is comparable to the covered distance. In order to study the waves with an asymmetric profile, which was deformed as a result of the manifestation of nonlinear effects, it is necessary to use numerical methods. 


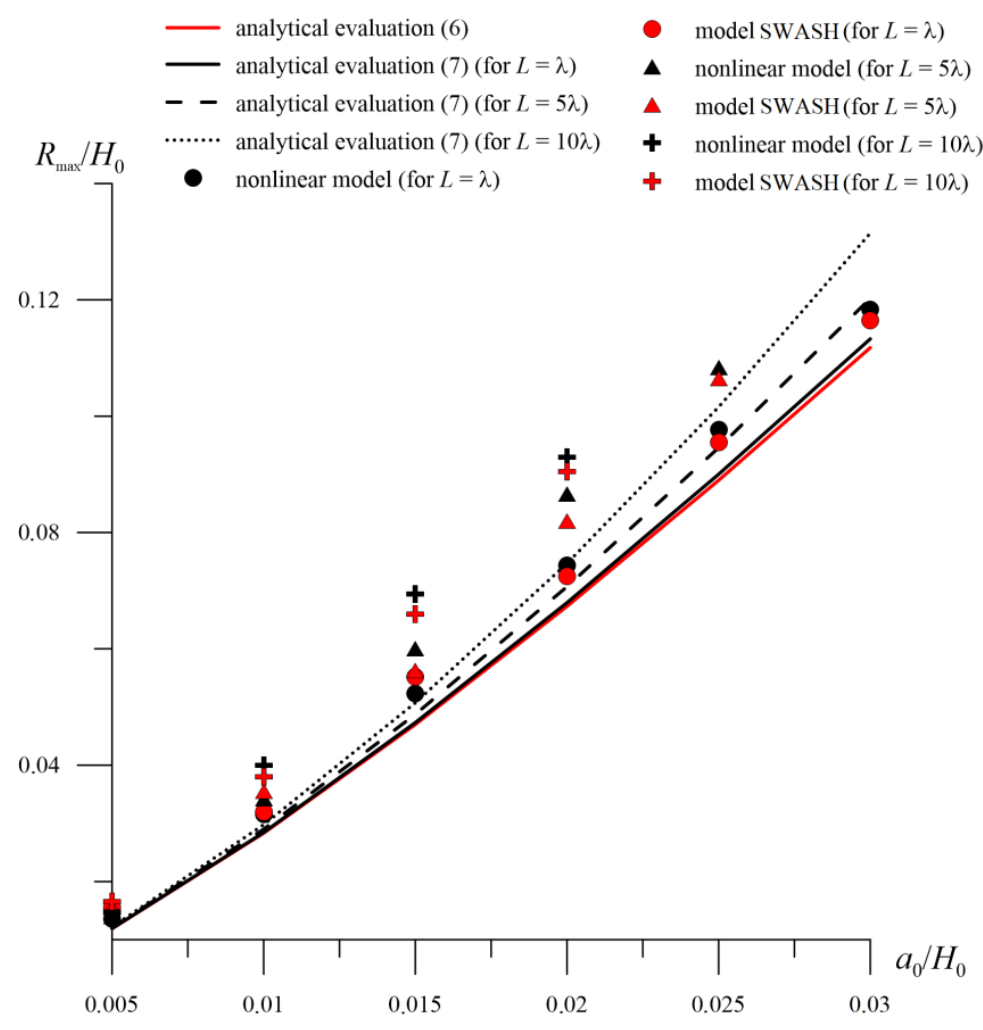

F i g. 6. Dependences of the maximum run-ups of the unbroken solitary waves. The basin depth $H_{0}=$ $=20 \mathrm{~m}$, the bottom slope $\operatorname{tg} \alpha=0.1$

\section{Conclusion}

Using nonlinear models of long waves, a series of numerical experiments was carried out to study the deformation of a solitary wave in a basin of shallow depth and subsequent run-up onto a gentle coast. The curves obtained for the soliton profiles in different models are in good agreement. It is shown that with an increase in the length of a constant depth section of the basin, the wave profile is deformed as a result of an increase in the steepness of the wave front slope. This, in its turn, has a significant effect on the increase in the run-up when the wave rushes to the shore. It was found that the undeformed waves when they roll onto the shore are amplified by 3.2 times with an initial amplitude of $0.2 \mathrm{~m}$, and the maximum deformed waves - by 4.4 times. With an amplitude of $0.3 \mathrm{~m}$, the ratios of the overshoots of undeformed and deformed waves to their amplitude were 3.5 and 4.5-4.6, and with an amplitude of $0.5 \mathrm{~m}-3.9$ and 4.2-4.3, respectively. With an increase in the initial amplitude of a solitary wave, the length of the path is reduced and the breaking occurs faster.

The calculated values of the maximum run-up onto the coast of undeformed waves, the length of which is comparable to the distance covered, are close to the estimates obtained analytically. For the waves approaching the shore with a steep front slope, the splash heights increase with a rise of the initial wave 
amplitude. In order to estimate the height of such waves' run-up to the coast, it is necessary to use numerical models.

\section{REFERENCES}

1. Kononkova, G.E. and Pokazeev, K.V., 1985. [Dynamics of Sea Waves]. Moscow: Publishing house of Moscow State University, 298 p. (in Russian).

2. Shelkovnikov, N.K., 2012. Soliton Version of Formation of Freak Waves in the Ocean. Morskoy Gidrofizicheskiy Zhurnal, (5), pp. $34-42$ (in Russian).

3. Squire, V.A., Kovalev, P.D. and Kovalev, D.P., 2021. Soliton-like Waves in the Vicinity of the Southern Kuril Islands. Physical Oceanography, 28(1), pp. 3-19. doi:10.22449/02337584-2021-1-3-19

4. Didenkulova, I.I. and Pelinovsky, E.N., 2019. Run-Up of a Nonlinear Monochromatic Wave on a Plane Beach in Presence of a Tide. Oceanology, 59(4), pp. 478-481. https://doi.org/10.1134/S0001437019040027

5. Carrier, G. and Greenspan, H., 1958. Water Waves of Finite Amplitude on a Sloping Beach. Journal of Fluid Mechanics, 4(1), pp. 97-109. https://doi.org/10.1017/S0022112058000331

6. Pedersen, G. and Gjevik, B., 1983. Run-up of Solitary Waves. Journal of Fluid Mechanics, 135, pp. 283-299. https://doi.org/10.1017/S0022112083003080

7. Synolakis, C.E., 1987. The Run-up of Solitary Waves. Journal of Fluid Mechanics, 185, pp. 523-545. https://doi.org/10.1017/S002211208700329X

8. Li, Y. and Raichlen, F., 2001. Solitary Wave Runup on Plane Slopes. Journal of Waterway, Port, Coastal, and Ocean Engineering, 127(1), pp. 33-44. https://doi.org/10.1061/(ASCE)0733-950X(2001)127:1(33)

9. Dotsenko, S.F. and Sannikova, N.K.V., 2013. Characteristics of the Solitary Surface Wave Runup on a Sloping Beach. Reports of the National Academy of Sciences of Ukraine, (2), pp. 86-91 (in Russian).

10. Rodin, A.A., Zemlyanikin, A.Y., Likhodeev, N.M., Kurkin, A.A. and Rodina, N.A., 2017. Laboratory and Numerical Study of the Wave Run-Up on the Flat Slope. In: PSU, 2017. NonEquilibrium Processes in Continuous Media. Materials of the international symposium. Vol. 2, pp. 166-168 (in Russian).

11. Nikishov, V.I., Selezov, I.T. and Khomitsky, V.V., 2011. Interaction of Surface and Internal Solitary Waves with Slopes. Applied Hydromechanics, 13(2), pp. 51-63. Available at: http://dspace.nbuv.gov.ua/handle/123456789/116297 [Accessed: 20 November 2021] (in Russian).

12. Maximov, V.V., Nudner, I.S., Lebedev, V.V., Semenov, K.K., Kshevetskii, S.P. and Amosov, A.A., 2017. Interaction of Tsunami Waves with Sloping Structures. Fundamentalnaya i Prikladnaya Gidrofizika, 10(4), pp. 53-60. doi:10.7868/S2073667317040050 (in Russian).

13. Didenkulova, I.I., Zahibo, N., Kurkin, A.A., Levin, B.V., Pelinovsky, E.N. and Soomere, T., 2006. Runup of Nonlinearly Deformed Waves on a Coast. Doklady Earth Sciences, 411(1), pp. 1241-1243. https://doi.org/10.1134/S1028334X06080186

14. Bazykina, A.Yu. and Fomin, V.V., 2017. Run-Up Characteristics of the Solitary Waves on the Coast in the Bays with Different Cross-Section Configurations. Ecological Safety of Coastal and Shelf Zones of Sea, (4), pp. 30-38 (in Russian).

15. Didenkulova, I.I., 2009. New Trends in the Analytical Theory of Long Sea Wave Runup. In: E. Quak and T. Soomere, eds., 2009. Applied Wave Mathematics. Berlin, Heidelberg: Springer, pp. 265-296. https://doi.org/10.1007/978-3-642-00585-5_14

16. Abdalazeez, A.A., Didenkulova, I.I. and Dutykh, D., 2019. Nonlinear Deformation and Runup of Single Tsunami Waves of Positive Polarity: Numerical Simulations and Analytical Predictions. Natural Hazards and Earth System Sciences, 19(12), pp. 2905-2913. https://doi.org/10.5194/nhess-19-2905-2019

17. Lamb, H., 1932. Hydrodynamics. Cambridge University Press, 738 p. 
18. Kowalik, Z., 2001. Basic Relations between Tsunami Calculations and Their Physics. Science of Tsunami Hazards, 19(2), pp. 99-115.

About the authors:

Aleksandra Yu. Belokon, Junior Research Associate, Marine Hydrophysical Institute of RAS (2 Kapitanskaya St., Sevastopol, 299011, Russian Federation), SPIN-code: 5633-9877, ORCID ID: 0000 -0002-1299-0983, ResearcherID: M-6839-2018, aleksa.44.33@gmail.com

Sergey Yu. Mikhailichenko, Junior Research Associate, Marine Hydrophysical Institute of RAS (2 Kapitanskaya St., Sevastopol, 299011, Russian Federation), SPIN-code: 7351-9338, ORCID ID: 0000 -0001-6332-1395, ResearcherID: M5788-2018, liham1984@rambler.ru

Contribution of the co-authors:

Aleksandra Yu. Belokon - the problem setting out and formulation, development and debugging of the computer program for solving the problem, analysis of the results, preparation of graphic materials, writing and editing of the article

Sergey Yu. Mikhailichenko - development and debugging of the computer program for solving the problem, discussion of the results, writing and editing of the article

All the authors have read and approved the final manuscript.

The authors declare that they have no conflict of interest. 